\title{
Determinants of First-line Antiretroviral Treatment Failure Among Patients on Antiretroviral Therapy in Public Hospitals Jimma, Southwest Ethiopia a Case-Control Study
}

\author{
Amrachu Bekele Asfaw ${ }^{1, ~ *, ~ A d e r a j e w ~ N i g u s i e ~}{ }^{2}$, Tamirat Shewanow ${ }^{3}$, Esayas Kebede Gudina ${ }^{4}$, \\ Masrie Getnet ${ }^{3}$, Demuma Amdisa ${ }^{5}$, Tefera Belachew Lemma ${ }^{2}$, Lelisa Sena Dadi ${ }^{3}$ \\ ${ }^{1}$ Public Health Department, Disease Prevention and Control Unit Mizan Teferi, Southwest, Ethiopia \\ ${ }^{2}$ Department of Population and Family Health, Jimma University, Jimma, Ethiopia \\ ${ }^{3}$ Department of Epidemiology, Jimma University, Jimma, Ethiopia \\ ${ }^{4}$ Departments of Internal Medicine, Jimma University, Jimma, Ethiopia \\ ${ }^{5}$ Department of Health Behaviour and Society, Jimma University, Jimma, Ethiopia
}

\section{Email address:}

amribekele@gmail.com (A. B. Asfaw), aderajewnu@gmail.com (A. Nigusie), babiynos@gmail.com (T. Shewanow), esakgd@gmail.com (E. K. Gudina), masriegetnet16.biostat@gmail.com (M. Getnet), amdisademuma@gmail.com (D. Amdisa), teferabelachew@gmail.com (T. B. Lemma), lelisajitu@gmail.com(L. S. Dadi)

*Corresponding author

\section{To cite this article:}

Amrachu Bekele Asfaw, Aderajew Nigusie, Tamirat Shewanow, Esayas Kebede Gudina, Masrie Getnet, Demuma Amdisa, Tefera Belachew Lemma, Lelisa Sena Dadi. Determinants of First-line Antiretroviral Treatment Failure Among Patients on Antiretroviral Therapy in Public Hospitals Jimma, Southwest Ethiopia a Case-Control Study. Rehabilitation Science. Vol. 4, No. 2, 2019, pp. 13-24. doi: 10.11648/j.rs.20190402.11

Received: March 16, 2019; Accepted: October 14, 2019; Published: October 23, 2019

\begin{abstract}
Highly Active Antiretroviral Therapy (HAART) has substantially declined morbidity and mortality related to Human immunodeficiency virus/Acquired immunodeficiency syndrome (HIV/AIDS). Despite this fact, first-line ART failure has emerged as a growing concern. However, factors associated with first-line ART failure are not well empathized and studied. Hence, we aimed to identify the determinants of first-line ART failure among patients attending ART in Public Hospitals Jimma, Southwest Ethiopia. A case-control study was conducted in March 2018 on a sample of 384 (288 controls and 96 cases) adult people living with HIV/AIDS (PLWHA). Cases were HIV patients aged 15 years or older who were on first-line ART regimens with documented therapeutic failure. Controls were HIV patients aged 15 years or older who were on first-line ART regimen but without evidence of therapeutic failure. Data were extracted from electronic databases and supplemented by data collected through interviewer-administered questionnaires. Bivariate and multivariate logistic regression analyses were used. Adjusted odds ratios and $95 \%$ confidence intervals were used to report independently associated factors at $\mathrm{P}$-value $<0.05$. In this study, higher odds of first-line ART failure was experienced among urban residents (AOR:2.2; 95\%CI: 1.1, 3.6), smokers (AOR:5.9; 95\%CI:3.2, 10.8), Khat users (AOR:2.2; 95\%CI:1.3,3.7), poor treatment adherents (AOR:2.2; 95\%CI: 1.1,4.5), tuberculosis coinfection (AOR:3.9; 95\%CI:2.2, 6.8), prior exposure to ART (AOR:3.8; 95\%CI:1.7, 8.1), zidovudine based regimen (AOR:4.8; 95\%CI: 2.5,9.0) and longer duration on ART more than 73 months (AOR:1.9; 95\%CI:1.2, 3.3). This study evidenced that being an urban resident, TB co-infection, poor medication adherence, and zidovudine-based regiment were positively and independently associated with first-line ART failure. Thus, the focus should be given assessment and management of medication compliance for urban residents and longer duration ART users. Assessment and management of substance use disorders are highly recommended besides ARV medication refills. Attention should be given enhanced adherence counseling and peer support to improve adherence. Early screening and management of tuberculosis infection should be strengthened. It is advisable to initiate ART with the recommended TDF-based first-line ART regimens instead of AZT-based.
\end{abstract}

Keywords: Determinants, First-Line ART Failure, HIV/AIDS, Jimma Zone, Public Hospitals 


\section{Introduction}

Globally, it is estimated that 36.9 million people were living with HIV during 2017. Sub-Saharan Africa (SSA) remains significantly affected, accounting for $69.5 \%$ of the people living with HIV (PLWHA). Ethiopia is among the SSA countries most affected by HIV/AIDS with an estimated 710,000 people living with HIV in 2016 [1, 2]. The introduction of highly active antiretroviral therapy (HAART) was a critical milestone in the history of HIV disease resulting in a dramatic reduction of morbidity and mortality, and improvement in the quality of life of PLWHA [3-5].

Despite the considerable reduction of morbidity and mortality due to HIV/AIDS after the start of HAART, a significant proportion of patients fail to attain the intended target of viral load suppression to therapy and many of them experience clinical deterioration attributable to ART failure. Treatment failure occurs when a combination of the ARV regimen fails to control HIV infection. This could be virologic, immunologic and/or clinical failure [6, 7].

Virologic failure occurs when the plasma viral load is 1000 copies $/ \mathrm{ml}$ and above in two consecutive measurements within the three-month interval with adherence support after at least six months of using ART. Immunologic failure occurs when CD4 cell count is lowered to 250 cells $/ \mathrm{mm}^{3}$ or below following clinical failure, or persistent CD4 cell level below 100 cells $/ \mathrm{mm}^{3}$ [8]. Clinical failure is the occurrence of new or recurrent WHO clinical stage four conditions [8]. Currently, the WHO recommends viral load testing as the preferred monitoring approach to diagnose and confirm ART treatment failure; however, targeted viral load monitoring or CD4 cell count and clinical monitoring should be used to diagnose treatment failure in settings where viral load test is not routinely available [5].

ART failure is a major challenge to HIV/AIDS management in resource-limited settings including Ethiopia where the diagnosis and management of ART failure is a key problem [9]. The documented findings showed that Virologic and Immunologic failure was $23 \%$ and $21.6 \%$ respectively among PLWHA who were on ART as reported from Vietnam and India $[9,10]$. In SSA countries, rates of first-line ART failure vary between $11.1 \%$ and $24 \%$ [11]. Studies showed that East Africa has faced a high prevalence of immunologic failure which ranges from $8 \%$ to $57 \%$; studies from South Africa indicated that $19 \%$ of PLWHA had developed virologic and immunologic failure $[12,13]$.

In Ethiopia, first-line ART treatment failure is $19.8 \%$ (immunologic $15 \%$, clinical $6.3 \%$, and virologic failure $1.3 \%$ ) among adult PLWHA who are on ART according to the report from private health facilities in Addis Ababa [14]; Immunologic and clinical failure was $19.7 \%$ and $19.5 \%$ respectively as reported from Southwest Ethiopia [15]. Documented findings from the University of Gondar referral hospital, Debremarkos, St. Luke and Tulubolo hospitals showed that immunologic failures were $22.2 \%, 21 \%$, and $6.8 \%$, respectively [16-18].
Previous studies reported that various factors including being male gender [19], illiterate [20], widower [21], poor adherence and treatment interruption [22-25], WHO clinical stage III and/or IV [24-26], low CD4 cell at ART initiation $[6,19,26,27]$, presence of opportunistic infections [6, 24, 25], low BMI [6, 28], taking ARV regimen before ART initiation [22, 24], taking Stavudine and Zidovudine (AZT) based regimen [29], TB co- infection [27, 30, 31], substance use [32-34] and longer duration on ART [3, 20, 26, 35] were significantly associated with first line ART failure.

Currently, first-line ART failure is a major challenge on viral load suppression treatment targets and impairs the quality life of PLWHA in resource-limited settings including Ethiopia [36]. Hence investigating and managing determinant factors of first-line ART treatment failure is very important to achieve the viral load suppression treatment target and sustain the quality of life PLWHA. However, there is limited evidence on the determinant factors of first-line antiretroviral treatment failure among PLWHA who are on first-line HAART in Ethiopia and the study area. Therefore, this study aimed to identify the determinant factors of firstline antiretroviral treatment failure among patients who were on first-line ART in public hospitals of Jimma Zone, Southwest Ethiopia.

\section{Methods}

\subsection{Study Design and Setting}

A hospital-based case-control study was conducted from March 1 to 26/ 2018 in public hospitals of Jimma Zone, Southwest Ethiopia; namely Jimma University Specialized Hospital (JUSH), Shenen Gibe Hospital, Agaro Hospital, and Limu Genet Hospital. Jimma zone is one of the administrative zones in Oromia Regional State, located at $352 \mathrm{~km}$ from Addis Ababa. According to the zonal health department information, the zone had an estimated total population of 3,324,912 in the year 2017. The hospitals have been providing ART services for a long period and have maintained data of the patients in the electronic medical record database. There were 11,186 PLWHA taking HIV chronic care services during the data collection period. The study hospitals provide both first and second-line ART. However, viral load testing service for HIV care has been available only in JUSH since 2015 .

\subsection{Study Participants, Sample Size Determination and Sampling Technique}

All PLWHA adults aged 15 years or older who had to follow up with HAART between January 2004 and December 2017 were considered to be the source population of the study. A Case was defined to be HIV patients aged 15 years or older who were on HAART between January 2004 to December 2017 and documented to have first-line ART treatment failure either virologic, immunologic or clinical in the study hospitals. Controls were patients aged 15 years or 
older who were on HAART between January 2004 to December 2017 but who did not faile first-line ART treatment failure. PLWHA who were stayed on HAART for less than six months and who had incomplete relevant clinical data were excluded from the study.

Epi Info ${ }^{\mathrm{TM}} 7$ StatCalc was used to determine sample size by considering the significant determinant factor of first-line ART failure Proportion of controls and cases age $<35$ years exposure status of virologic failure were $47.7 \%$ and $64.7 \%$ respectively taken from a study finding from a previous study [35] using margin of error of $5 \%$ and power $80 \%$. Therefore, the total sample size was estimated to be 384 (288 controls and 96 cases; considering three to one controls to case ratio). There were 102 documented PLWHA first-line ART treatment failure; however, six of them were excluded because of incomplete relevant clinical data from their medical record cards whereas all the remaining 96 were included in the study without sampling because of their relatively small number. The desired number of controls $(\mathrm{n}=$ 288) who fulfilled the inclusion criteria were randomly selected using computer-generated random numbers based on the unique ART identification number. Proportion to sample size allocation was used to allocate the sample for each hospital.

Table 1. Calculated sample size for study on determinants of first-line antiretroviral treatment failure among PLWHA on HAART, at Jimma Zone Public Hospitals, South West Ethiopia 2018.

\begin{tabular}{llllll}
\hline Variables & $\begin{array}{l}\text { The ratio of Control to } \\
\text { Cases }\end{array}$ & $\begin{array}{l}\text { \% of controls } \\
\text { exposed }\end{array}$ & OR & \% of cases exposed & Total sample size \\
\hline Age $<35$ years (35) & 3 & 47.7 & 2.01 & 64.74 & $384(288: 96)$ \\
Snemployment (3) & 3 & 30 & 2.48 & 51.5 & $228(176: 59)$ \\
low base line BMI (3) & 3 & 45 & 2.08 & 63 & $348(261: 87)$ \\
Baseline CD4 count & 3 & 48.4 & 4.77 & 81.7 & $100(75: 25)$ \\
$\begin{array}{l}<\text { 200cells } / \text { mm }^{3}(35) \\
\text { Poor adherence (35) }\end{array}$ & 3 & 7.8 & 11.67 & 49.7 & $52(39: 13)$ \\
\hline
\end{tabular}

\subsection{Data Collection Tools and Procedure}

The sources of data for this study were both secondary and primary data. Secondary data were extracted from the electronic database using a structured checklist. Primary data was obtained using a structured interviewer-administered questionnaire. Data collection tools were prepared by adapting and contextualizing the WHO standard tool and Federal Ministry of Health of Ethiopia ART guideline as well as by reviewing related literature $[37,38]$. Sociodemographic profiles, substance use, treatment support, HIV result disclosure status, wealth index status and missed follow-up appointment was included in structured interviewer-administered questionnaires whereas Body mass index (BMI), Hemoglobin level, opportunistic infection, TB coinfection, functional status, HIV test entry, and type of ARV drugs were included in the checklist.

Ten nurses (six data collectors and four supervisors) who have experience in HIV care were recruited for data collection. The primary data was collected on the day of scheduled clinic visits for all cases and randomly selected controls. Participants, who were not available at the time of scheduled clinic visits were traced using the phone number and home address available on the card and interviewed on the consecutive days of the study period. For the secondary data, data collectors from ART clinics were accessed and reviewed electronic databases and patient charts.

\subsection{Study Variables}

The dependent variable was the occurrence of first-line antiretroviral treatment failure. In this study, first-line antiretroviral treatment failure was defined as the fulfillment of either virologic, immunologic or clinical failure criteria set by WHO. This was determined by reviewing whether the patient was diagnosed with first-line ART failure or not on his/her medical record. The independent variables include: socio-demographic and economic variables (age, residence, sex, religion, ethnicity, marital status, household relative wealth index, HIV disclosure status, missed clinic appointment, educational status, employment status), substance use (smoking, Khat chewing and alcohol use), adherence status (good, poor or fair), BMI, hematologic level, opportunistic infection, TB coinfection, functional status, HIV test entry, type of ARVs and treatment support history.

\subsection{Operational Definitions}

Khat (Catha edulis) is a leaf cultivated in Kenya, Yemen, and Ethiopia. It has alkaloids cathine and cathinone which have amphetamine-like properties and has been used for centuries in many African countries for its euphoric effect and as a sanctioned cultural practice. Khat has been associated with various health effects in the long-term, including oesophagitis, gastritis, duodenal ulcer, hepatic cirrhosis, autoimmune hepatitis, migraine, cerebral hemorrhage, pulmonary edema, and myocardial infarction when it is used frequently [39].

\subsection{Data Quality Assurance, Processing, and Statistical Analysis}

Data collectors and supervisors were trained on data collection tools before the commencement of data collection. Collected data entered into Epi Data version 3.1 and exported to SPSS version 23 for analysis. Simple frequencies were carried out to check data cleanness. Descriptive statistics including median and interquartile range (IQR) values and frequencies were used to summarize participant's characteristics in each group. Dimension reduction using factorial analysis by a method of principal components was 
employed to assess an individual's wealth index status. Assumptions of principal component analysis such as a measure of sampling adequacy (MSA) less than 0.5 , overall Kaiser-Meyer-Olkin (KMO) value less than 0.5 , communality value less than 0.5 and complex structure were checked for each variable. Variables with $\mathrm{p}$-value $<0.25$ at bivariate analysis were taken to multivariate logistic regression analysis. The multivariable model was fitted to identify the independent determinants of first-line ART failure using a backward stepwise removal method. The model fitness was checked by Hosmer-Lemeshow goodness of fit tests (chi-square at 6.57 and $p$-value $=0.583$ ). Finally, variables that had significant associations with first-line ART failure were identified and reported based on the adjusted odds ratio (AOR) with corresponding $95 \% \mathrm{CI}$ at $\mathrm{p}$-value $<0.05$.

\section{Results}

\subsection{Socio-demographic Characteristics of the Study Participants}

In this study, a total of $36(9.4 \%), 31(8.1 \%)$ and 29 (7.6\%) participants developed virologic, immunologic and clinical failure respectively. The median age at the time of ART initiation for cases and controls was 28 (IQRs 25-32) and 27 (IQRs23-33), respectively. Female participants were $53(55.2 \%)$ and $181(62.8 \%)$ among cases and controls, respectively [Table 2].

Table 2. Sociodemographic Characteristics of PLWHA $\geq 15$ years, who have had a follow-up at selected public hospitals of Jimma Zone, Southwest Ethiopia, 2004-2017.

\begin{tabular}{|c|c|c|c|}
\hline Variable & Categories & Cases n (\%) & Controls n (\%) \\
\hline \multirow{3}{*}{ Age, years } & Median & 28 (IQR 25-32) & 27 (IQR 23-33) \\
\hline & $<28$ years & $41(42.7)$ & $149(51.7)$ \\
\hline & $\geq 28$ years & $55(57.3)$ & $139(48.3)$ \\
\hline \multirow{2}{*}{ Sex } & Male & $43(44.8)$ & $107(37.2)$ \\
\hline & Female & $53(55.2)$ & $181(62.8)$ \\
\hline \multirow{3}{*}{ Marital Status } & Married & $57(59.4)$ & $174(60.4)$ \\
\hline & Single & $6(6.3)$ & $30(10.4)$ \\
\hline & Widowed & $11(11.5)$ & $40(13.9)$ \\
\hline \multirow{6}{*}{$\begin{array}{l}\text { Occupation } \\
\text { Status }\end{array}$} & Government employee & $20(20.8)$ & $83(28.8)$ \\
\hline & Private business & $52(54.2)$ & $116(40.3)$ \\
\hline & Student & $4(4.2)$ & $8(2.8)$ \\
\hline & Unemployed & $3(3.1)$ & $11(3.8)$ \\
\hline & Housewife & $7(7.3)$ & $45(15.6)$ \\
\hline & Farmer & $10(10.4)$ & $25(8.7)$ \\
\hline \multirow{4}{*}{$\begin{array}{l}\text { Wealth } \\
\text { Index }\end{array}$} & Second Quintile & $27(28.1)$ & $67(23.3)$ \\
\hline & Third Quintile & $14(14.6)$ & $64(22.2)$ \\
\hline & Fourth Quintile & $19(19.8)$ & $56(19.4)$ \\
\hline & Fifth Quintile & $20(20.8)$ & $57(19.8)$ \\
\hline HIV result disclosure & Yes & $74(77.1)$ & $218(75.7)$ \\
\hline Status & No & $22(22.9)$ & $70(24.3)$ \\
\hline Missed & Yes & $52(54.2)$ & $126(43.8)$ \\
\hline Appointment & No & $44(45.8)$ & $162(56.2)$ \\
\hline \multirow{2}{*}{ Religion } & Christian & $57(59.3)$ & $148(51.4)$ \\
\hline & Muslim & $39(40.6)$ & $140(48.6)$ \\
\hline \multirow{4}{*}{ Ethnicity } & Oromo & $48(50.0)$ & $163(56.6)$ \\
\hline & Amhara & $22(22.9)$ & $52(18.1)$ \\
\hline & Kaffa & $15(15.6)$ & $29(10.1)$ \\
\hline & Other $^{\mathrm{a}}$ & $11(11.5)$ & $34(15.2)$ \\
\hline Variables & Categories & Cases n (\%) & Controls n (\%) \\
\hline \multirow{2}{*}{ Educational status } & Secondary education & $19(19.8)$ & $59(20.5)$ \\
\hline & College and above & $15(15.6)$ & $42(14.6)$ \\
\hline \multirow{2}{*}{ Residence } & Urban & $77(80.2)$ & $186(64.6)$ \\
\hline & Rural & $19(19.8)$ & $102(35.4)$ \\
\hline
\end{tabular}

a: Gurage, Dawro, and Tigre

\subsection{Substance Use Characteristics}

Among the study participants, $35(36.5 \%)$ cases and 52 (18.1\%) controls had history of smoking, 24 (25.0\%) cases and 69 (24.0) controls had history of alcohol use whereas 44 (45.8\%) of cases and 91 (31.6\%) of controls had a history of Khat use [Table 3]. 
Table 3. Behavioral related information of PLWHA $\geq 15$ years, who have had a follow-up at selected public hospitals Jimma: $2004-2017$.

\begin{tabular}{|c|c|c|c|}
\hline Variable & Categories & Cases n (\%) & Controls n (\%) \\
\hline \multirow{2}{*}{ Smoking status } & Yes & $35(36.5)$ & $52(18.1)$ \\
\hline & No & $61(63.5)$ & $236(81.9)$ \\
\hline \multirow{2}{*}{ Alcohol use } & Yes & $24(25.0)$ & $69(24.0)$ \\
\hline & No & $72(75.0)$ & $219(76.0)$ \\
\hline \multirow{2}{*}{ Khat use } & Yes & $44(45.8)$ & $91(31.6)$ \\
\hline & No & $52(54.2)$ & $197(68.4)$ \\
\hline \multirow{2}{*}{ Frequency of using tobacco products } & Never & $61(63.5)$ & $236(81.9)$ \\
\hline & Once/twice a Week & $22(22.9)$ & $36(12.5)$ \\
\hline \multirow{3}{*}{ Frequency of using Alcoholic beverages } & Never & $72(75.0)$ & $220(76.4)$ \\
\hline & Daily & $8(8.3)$ & $46(16.0)$ \\
\hline & Once/twice a Week & $16(16.7)$ & $22(7.6)$ \\
\hline \multirow{3}{*}{ Frequency of using Khat } & Never & $52(54.2)$ & $197(68.4)$ \\
\hline & Daily & $40(41.7)$ & $57(19.8)$ \\
\hline & Once/twice a Week & $4(4.1)$ & $(11.8)$ \\
\hline
\end{tabular}

\subsection{Adherence and Antiretroviral Drug-related Characteristics}

The study revealed that $70(72.9 \%)$ and $261(90.6 \%)$ cases and controls had good baseline adherence to ARVs, respectively. Majority 90 (93.8\%) cases and 282 (97.9\%) controls have good current adherence to ARVs. Among the cases, $30(31.3 \%)$ and $38(39.6 \%)$ of them used TDF-3TC-
EFV and AZT-3TC-NVP initial ARV regiment, respectively. Whereas $154(53.5 \%)$ and 35 (12.2\%) of controls used TDF3TC-EFV and AZT-3TC-NVP initial ARV regiment, respectively. $61(63.5 \%)$ cases and $132(45.8 \%)$ of controls were on HAART for more than the median duration of 73 months [Table 4].

Table 4. Antiretroviral medication-related information of PLWHA $\geq 15$ years, who have had a follow-up at selected public hospitals Jimma: 2004-2017.

\begin{tabular}{llll}
\hline Variable & Categories & Cases n (\%) & Controls n (\%) \\
\hline \multirow{2}{*}{ Baseline adherence to treatment } & Good & $70(72.9)$ & $261(90.6)$ \\
& Fair/poor & $26(27.1)$ & $27(9.4)$ \\
Current adherence to treatment & Good & $90(93.8)$ & $282(97.9)$ \\
& Fair/poor & $6(6.3)$ & $6(2.1)$ \\
Prior ARV exposure & Yes & $32(33.3)$ & $20(6.9)$ \\
& No & $64(66.7)$ & $268(93.1)$ \\
& TDF-3TC-EFV & $30(31.3)$ & $154(53.5)$ \\
Initial ARV regimen & D4T-3TC-NVP & $13(13.5)$ & $52(18.1)$ \\
& AZT-3TC-NVP & $38(39.6)$ & $35(12.2)$ \\
& D4T-3TC-EFV & $4(4.2)$ & $21(7.3)$ \\
& AZT-3TC-EFV & $7(7.3)$ & $13(4.5)$ \\
Duration on HAART & TDF-3TC-NVP & $4(4.2)$ & $13(4.5)$ \\
& Median & $84.5(60-103.8)$ & $67.5(35-108)$ \\
\hline
\end{tabular}

\subsection{Clinical Characteristics of the Study Participants}

Regarding opportunistic infections, $52(54.2 \%)$ cases and $174(60.4 \%)$ controls had a history of baseline opportunistic infections. $53(55.2 \%)$ of cases and $77(26.7 \%)$ of controls had TB coinfection at the time of ART initiation. Concerning BMI, $45(46.9 \%)$ of the cases had baseline median BMI $<$ $19.3 \mathrm{~kg} / \mathrm{m}^{2}$, whereas $147(51.0 \%)$ of controls had baseline median $\mathrm{BMI}<19.3 \mathrm{~kg} / \mathrm{m}^{2}$ [Table 5].

Table 5. Clinical related information of PLWHA $\geq 15$ years, who have had a follow-up at selected public hospitals Jimma: $2004-2017$.

\begin{tabular}{|c|c|c|c|}
\hline Variable & Categories & Cases n (\%) & Controls n (\%) \\
\hline \multirow{4}{*}{ HIV testing entry } & VCT & $41(42.7)$ & $114(39.6)$ \\
\hline & PITC & $19(19.8)$ & $62(21.5)$ \\
\hline & PMTCT & $7(7.3)$ & $19(6.6)$ \\
\hline & Other $^{\mathrm{b}}$ & $29(30.2)$ & $93(32.3)$ \\
\hline \multirow{2}{*}{ Baseline opportunistic infection } & Yes & $52(54.2)$ & $174(60.4)$ \\
\hline & No & $44(45.8)$ & $114(39.6)$ \\
\hline Current opportunistic infection & Yes & $11(11.5)$ & $24(8.3)$ \\
\hline \multirow{2}{*}{ TB coinfection at ART initiation } & Yes & $53(55.2)$ & $77(26.7)$ \\
\hline & No & $43(44.8)$ & $211(73.3)$ \\
\hline \multirow{2}{*}{ Baseline functional status } & Working & $64(66.6)$ & $215(74.7)$ \\
\hline & Ambulatory & $26(27.1)$ & $62(21.5)$ \\
\hline
\end{tabular}




\begin{tabular}{llll}
\hline Variable & Categories & Cases n (\%) & Controls n (\%) \\
\hline & Bed ridden & $6(6.3)$ & $11(3.8)$ \\
& Working & $92(95.8)$ & $278(96.5)$ \\
Current functional status & Ambulatory & $3(3.1)$ & $9(3.1)$ \\
& Bed ridden & $1(1.0)$ & $1(0.3)$ \\
& Median & $18.9(\mathrm{IQR} 16.0-21.5)$ & $19.4(\mathrm{IQR} 17.6-21.8)$ \\
Baseline BMI kg/m ${ }^{2}$ & $<19.3 \mathrm{~kg} / \mathrm{m}^{2}$ & $45(46.9 \%)$ & $147(51.0 \%)$ \\
& $\geq 19.3 \mathrm{~kg} / \mathrm{m}^{2}$ & $51(53.1 \%)$ & $141(49.0 \%)$ \\
& Median & $21.6(\mathrm{IQR} 18.7-23.6)$ & $21.4(\mathrm{IQR} 19.4-24.0)$ \\
Current BMI kg/m & $<21.5 \mathrm{~kg} / \mathrm{m}^{2}$ & $51(53.1 \%)$ & $142(49.3 \%)$ \\
& $\geq 21.5 \mathrm{~kg} / \mathrm{m}^{2}$ & $45(46.9 \%)$ & $146(50.7 \%)$ \\
\hline
\end{tabular}

Notes: ${ }^{\mathrm{b}}$ on campaign session

\subsection{Hemoglobin Profile of the Study Participants}

In this study $41(42.7 \%)$ of cases and $91(31.6 \%)$ of controls had baseline median Hemoglobin level less than $12 \mathrm{~g} / \mathrm{dl} \quad(\mathrm{IQR}: 11-12.8)$ and 12g/dl (IQR:11.2-13.1), respectively whereas $55(57.3 \%)$ cases and 197 (68.4) had baseline median Hemoglobin level greater than or equal 12(IQR11-12.8) and 12(IQR11.2-13.1) respectively [Table $6]$.

Table 6. Haemoglobin Profile of PLWHA $\geq 15$ years, who have had a follow-up at selected public hospitals Jimma: 2004-2017

\begin{tabular}{llll}
\hline Variable & Categories & Cases n (\%) & Controls n (\%) \\
\hline Baseline & Median & $12(\mathrm{IQR} 11-12.8)$ & $12(\mathrm{IQR} 11.2-13.1)$ \\
hemoglobin status & $\geq 12 \mathrm{~g} / \mathrm{dl}$ & $55(57.3)$ & $197(68.4)$ \\
(median) & $<12 \mathrm{~g} / \mathrm{dl}$ & $41(42.7)$ & $91(31.6)$ \\
Current & Median & $13(\mathrm{IQR} 12.4-14.1)$ & $13(\mathrm{IQR} 12.5-14.1)$ \\
hemoglobin status & $\geq 13 \mathrm{~g} / \mathrm{dl}$ & $56(58.3)$ & $200(69.4)$ \\
(median) & $<13 \mathrm{~g} / \mathrm{dl}$ & $40(41.7)$ & $88(30.6)$ \\
\hline
\end{tabular}

\subsection{Treatment Support Characteristics of Study Participants}

Regarding treatment support, 47 (49\%) of cases and 182 (63.2\%) of controls had history of treatment support, of whom $21(21.9 \%)$ of cases and $80(27.8 \%)$ of controls had emotional support; $22(22.9 \%)$ of cases and $76(26.4 \%)$ of controls had physical support; $23(24.0 \%)$ of cases and 118 $(41.0 \%)$ of controls have got the supports from their family whereas $19(19.8 \%)$ of cases and $39(13.5 \%)$ of controls obtained the supports from governmental organizations.

\subsection{Determinant Factors Associated with First-line ART Treatment Failure}

The bivariate logistic regression analysis indicated that age, sex, marital status, occupation status, missed follow up appointment, residence, smoking, Khat use, baseline, and current adherence status, prior exposure to ARV dose, AZT and EFV based medication, longer duration on HAART, baseline functional status, TB coinfection and baseline, and current hemoglobin level were associated with first-line ART failure. However, multivariate logistic regression analysis revealed that being urban resident, smoking, Khat use, poor baseline adherence status, TB coinfection at the time of ART initiation, prior exposure to ARV, taking AZT based first-line ARV regiment, median duration on HAART for $\geq 73$ months were found to be significantly associated with first-line ART treatment failure. However: age, sex, marital status, occupational status, missed follow up appointment, current adherence status, EFV based medication, baseline, and current hemoglobin level were not significantly associated with first-line ART treatment failure.

ART users who resided urban were two times more likely to develop first-line ART failure than those reside rural (AOR:2.2; 95\% CI:1.1, 3.6). ART users who had a smoking history were 5.9 times more likely to develop first-line ART failure than those who did not (AOR:5.9; 95\% CI: 3.2,10.8). ART users who had a history of Khat use were two times more likely to develop first-line ART failure than those who did not (AOR: $2.2 ; 95 \%$ CI:1.3, 3.7).

ART users with poor medication adherence were two times more likely to develop first-line ART failure than patients with good adherence (AOR:2.2; 95\%CI:1.1,4.5). ART users with baseline TB coinfection were 3.9 times as likely to develop first-line ART treatment failure as those not coinfected (AOR: 3.9; 95\% CI: 2.2, 6.8). ART users with prior exposure to ART were 3.8 times more likely to develop first-line ART failure than those non-exposed (AOR:3.8; 95\% CI:1.7, 8.1). ART users with AZT based medication were 4.8 times as likely to develop first-line ART failure as ART users with TDF based medication (AOR:4.8; 95\% CI: $2.5,8.0)$. ART users stayed on ART for a median duration $>=73$ months were two times more likely to develop first-line ART failure than those who took treatment for $<73$ months (AOR:1.9; 95\% CI: 1.2, 3.3) [Table 7].

Table 7. Determinants of First-line ART failure among PLWHA > 15 years, who had a follow-up at selected public hospitals Jimma: $2004-2017$.

\begin{tabular}{|c|c|c|c|c|c|c|}
\hline \multirow{2}{*}{ Variables } & \multicolumn{2}{|c|}{ First-line ART Failure } & \multicolumn{2}{|c|}{ Bivariate Analysis } & \multicolumn{2}{|c|}{ Multivariate Analysis } \\
\hline & Cases n (\%) & Controls n (\%) & P-value & COR (95\%CI) & P-value & AOR (95\%CI) \\
\hline Baseline Median Age & 28 (IQR 25-32) & 27 (IQR 23-33) & & & & \\
\hline$>=28$ years & $55(57.3)$ & $139(48.3)$ & 0.126 & $1.4(0.9,2.3)$ & - & - \\
\hline$<28$ years & $41(42.7)$ & $149(51.7)$ & 1 & 1 & 1 & 1 \\
\hline Sex & & & & & & \\
\hline Male & $43(44.8)$ & $107(37.2)$ & 0.185 & $1.3(0.9,2.2)$ & - & - \\
\hline Female & $53(55.2)$ & $181(62.8)$ & 1 & 1 & 1 & 1 \\
\hline
\end{tabular}




\begin{tabular}{|c|c|c|c|c|c|c|}
\hline \multirow{2}{*}{ Variables } & \multicolumn{2}{|c|}{ First-line ART Failure } & \multicolumn{2}{|c|}{ Bivariate Analysis } & \multicolumn{2}{|c|}{ Multivariate Analysis } \\
\hline & Cases n (\%) & Controls n (\%) & P-value & COR (95\%CI) & P-value & AOR $(95 \% \mathrm{CI})$ \\
\hline \multicolumn{7}{|l|}{ Marital status } \\
\hline Married & $57(59.4)$ & $174(60.4)$ & 1 & 1 & 1 & 1 \\
\hline Single & $6(6.3)$ & $30(10.4)$ & 0.296 & $1.6(0.2,1.5)$ & - & - \\
\hline Divorced & $22(22.9)$ & $44(15.3)$ & 0.162 & $0.7(0.8,2.8)$ & - & - \\
\hline Widowed & $11(11.5)$ & $40(13.9)$ & 0.639 & $1.2(0.4,1.7)$ & - & - \\
\hline \multicolumn{7}{|l|}{ Occupation status } \\
\hline Government employee & $20(20.8)$ & $83(28.8)$ & 1 & 1 & 1 & 1 \\
\hline Private business & $52(54.2)$ & $116(40.3)$ & 0.038 & $0.5(1.0,3.4)$ & - & - \\
\hline Unemployed & $24(25)$ & $89(30.9)$ & 0.358 & $0.9(0.3,1.6)$ & - & - \\
\hline \multicolumn{7}{|l|}{ Wealth Index status** } \\
\hline Fifth quintile & $20(20.8)$ & $57(19.8)$ & 1 & 1 & 1 & 1 \\
\hline Fourth quintile & $19(19.8)$ & $56(19.4)$ & 0.928 & $1.0(0.5,2.0)$ & & \\
\hline Third quintile & $14(14.6)$ & $64(22.2)$ & 0.229 & $1.6(0.3,1.4)$ & & \\
\hline Second quintile & $27(28.1)$ & $67(23.3)$ & 0.689 & $0.9(0.6,2.3)$ & & \\
\hline First quintile & $16(16.7)$ & $44(15.3)$ & 0.927 & $1.0(0.5,2.2)$ & & \\
\hline \multicolumn{7}{|l|}{ HIV result disclosure Status** } \\
\hline Yes & $74(77.1)$ & $218(75.7)$ & 1 & 1 & 1 & 1 \\
\hline No & $22(22.9)$ & $70(24.3)$ & 0.783 & $1.1(0.6,1.9)$ & & \\
\hline \multirow{2}{*}{ Variables } & \multicolumn{2}{|c|}{ First-line ART Failure } & \multicolumn{2}{|c|}{ Bivariate Analysis } & \multicolumn{2}{|c|}{ Multivariate Analysis } \\
\hline & Cases n (\%) & Controls n (\%) & P-value & COR $(95 \% \mathrm{CI})$ & P-value & AOR (95\%CI) \\
\hline \multicolumn{7}{|l|}{ Missed follow up appointment } \\
\hline Yes & $52(54.2)$ & $126(43.8)$ & 0.077 & $1.5(0.9,2.4)$ & - & - \\
\hline No & $44(45.8)$ & $162(56.2)$ & 1 & 1 & 1 & 1 \\
\hline Educational status** & & & & & & \\
\hline No formal education & $19(19.8)$ & $39(13.5)$ & 0.450 & $1.3(0.6,3.0)$ & & \\
\hline Primary education & $43(44.8)$ & $148(51.4)$ & 0.552 & $0.8(0.4,1.6)$ & & \\
\hline Secondary education & $19(19.8)$ & $59(20.5)$ & 0.796 & $0.9(0.4,1.0)$ & & \\
\hline College and above & $15(15.6)$ & $42(14.6)$ & 1 & 1 & 1 & 1 \\
\hline Residence* & & & & & & \\
\hline Urban & $77(80.2)$ & $186(64.6)$ & 0.004 & $2.2(1.3,3.9)$ & 0.010 & $2.2(1.1,3.6)$ \\
\hline Rural & $19(19.8)$ & $102(35.4)$ & 1 & 1 & 1 & 1 \\
\hline Smoking* & & & & & & \\
\hline Yes & $35(36.5)$ & $52(18.1)$ & 0.000 & $2.6(1.6,4.4)$ & 0.000 & $5.9(3.2,10.8)$ \\
\hline No & $61(63.5)$ & $236(81.9)$ & 1 & 1 & 1 & 1 \\
\hline Alcohol use** & & & & & & \\
\hline Yes & $24(25.0)$ & $69(24.0)$ & 0.837 & $1.1(0.6,1.8)$ & - & - \\
\hline No & $72(75.0)$ & $219(76.0)$ & 1 & 1 & 1 & 1 \\
\hline Khat chewing* & & & & & & \\
\hline Yes & $44(45.8)$ & $91(31.6)$ & 0.011 & $1.8(1.1,2.9)$ & 0.002 & $2.2(1.3,3.7)$ \\
\hline No & $52(54.2)$ & $197(68.4)$ & 1 & 1 & 1 & 1 \\
\hline Variables & First-line ART Fail & ure & Bivariat & nalysis & Multivar & lysis \\
\hline Vartadoies & Cases n (\%) & Controls n (\%) & P-value & COR $(95 \% \mathrm{CI})$ & P-value & AOR (95\%CI) \\
\hline Baseline adherence status* & & & & & & \\
\hline Fair/poor & $26(27.1)$ & $27(9.4)$ & 0.000 & $3.6(1.0,6.5)$ & 0.037 & $2.2(1.1,4.5)$ \\
\hline Good & $70(72.9)$ & $261(90.6)$ & 1 & 1 & 1 & 1 \\
\hline Current adherence to treatment & & & & & & \\
\hline Fair/poor & $6(6.3)$ & $6(2.1)$ & 0.042 & $3.1(0.9,9.9)$ & - & - \\
\hline Good & $90(93.8)$ & $282(97.9)$ & 1 & 1 & 1 & 1 \\
\hline Prior ARV exposure* & & & & & & \\
\hline Yes & $32(33.3)$ & $20(6.9)$ & 0.000 & $6.7(3.6,12.5)$ & 0.001 & $3.8(1.7,8.1)$ \\
\hline No & $64(66.7)$ & $268(93.1)$ & & & & \\
\hline Initial first-line ARV regimen ( $\mathrm{T}$ & D4T, AZT) based* & & & & & \\
\hline AZT based & $45(46.9)$ & $48(16.7)$ & 0.000 & $4.6(2.7,7.0)$ & 0.000 & $4.8(2.5,8.0)$ \\
\hline D4T based & $17(17.7)$ & $73(25.3)$ & 0.682 & $1.14(0.6,2.2)$ & 0.793 & $0.9(0.4,1.9)$ \\
\hline TDF based & $34(35.4)$ & $167(58)$ & 1 & 1 & 1 & 1 \\
\hline Initial first line $\mathrm{ARV}$ regimen (E) & NVP) based & & & & & \\
\hline NVP based & $55(57.3)$ & $100(34.7)$ & 0.000 & $2.5(1.6,4.0)$ & - & - \\
\hline EFV based & $41(42.7)$ & $188(65.3)$ & 1 & 1 & 1 & 1 \\
\hline Duration on HAART (median)* & 84.5 (IQR60-103.8) & 67.5 (IQR35-108) & & & & \\
\hline$>=73$ months & $61(63.5)$ & $132(45.8)$ & 0.003 & $2.1(1.3,3.3)$ & 0.009 & $1.9(1.2,3.3)$ \\
\hline$<73$ months & $35(36.5)$ & $156(54.2)$ & 1 & 1 & 1 & 1 \\
\hline HIV test entry** & & & & & & \\
\hline PITC & $19(19.8)$ & $62(21.5)$ & 0.574 & $0.8(0.4,1.6)$ & & \\
\hline
\end{tabular}




\begin{tabular}{|c|c|c|c|c|c|c|}
\hline \multirow{2}{*}{ Variables } & \multicolumn{2}{|c|}{ First-line ART Failure } & \multicolumn{2}{|c|}{ Bivariate Analysis } & \multicolumn{2}{|c|}{ Multivariate Analysis } \\
\hline & Cases n (\%) & Controls n (\%) & P-value & COR $(95 \% \mathrm{CI})$ & P-value & AOR $(95 \% \mathrm{CI})$ \\
\hline РMTCT & $7(7.3)$ & $19(6.6)$ & 0.902 & $1.0(0.4,2.7)$ & & \\
\hline Other $^{b}$ & $29(30.2)$ & $93(32.3)$ & 0.594 & $0.9(0.5,1.5)$ & & \\
\hline VCT & $41(42.7)$ & $114(39.6)$ & 1 & 1 & 1 & 1 \\
\hline
\end{tabular}

\begin{tabular}{|c|c|c|c|c|c|c|}
\hline \multirow{2}{*}{ Variables } & \multicolumn{2}{|l|}{ First-line ART Failure } & \multicolumn{2}{|c|}{ Bivariate Analysis } & \multicolumn{2}{|c|}{ Multivariate Analysis } \\
\hline & Cases n (\%) & Controls n (\%) & P-value & COR $(95 \%$ CI $)$ & P-value & AOR $(95 \% C I)$ \\
\hline \multicolumn{7}{|l|}{ Baseline functional status } \\
\hline Working & $64(66.6)$ & $215(74.7)$ & 1 & 1 & 1 & 1 \\
\hline Ambulatory & $26(27.1)$ & $62(21.5)$ & 0.189 & $1.4(0.8,2.5)$ & - & - \\
\hline Bed ridden & $6(6.3)$ & $11(3.8)$ & 0.338 & $1.7(0.5,5.3)$ & - & - \\
\hline \multicolumn{7}{|l|}{ Current functional status** } \\
\hline Working & $92(95.8)$ & $278(96.5)$ & 1 & 1 & 1 & 1 \\
\hline Ambulatory & $3(3.1)$ & $9(3.1)$ & 0.789 & $0.8(0.2,3.2)$ & & \\
\hline Bed ridden & $1(1.0)$ & $1(0.3)$ & 0.665 & $1.9(0.1,38.1)$ & & \\
\hline \multicolumn{7}{|l|}{ TB coinfection at baseline* } \\
\hline Yes & $53(55.2)$ & $77(26.7)$ & 0.000 & $3.4(2.0,5.5)$ & 0.000 & $3.9(2.2,6.8)$ \\
\hline No & $43(44.8)$ & $211(73.3)$ & 1 & 1 & 1 & 1 \\
\hline \multicolumn{7}{|l|}{ Baseline opportunistic infection** } \\
\hline No & $44(45.8)$ & $114(39.6)$ & 1 & 1 & 1 & 1 \\
\hline Yes & $52(54.2)$ & $174(60.4)$ & 0.283 & $1.2(0.8,2.1)$ & & \\
\hline \multicolumn{7}{|l|}{ Current opportunistic infection** } \\
\hline Yes & $11(11.5)$ & $24(8.3)$ & 0.358 & $1.4(0.7,3.0)$ & & \\
\hline No & $85(88.5)$ & $264(91.7)$ & 1 & 1 & 1 & 1 \\
\hline Baseline BMI kg/m² (median)** & 18.9 (IQR 16.0-21.5) & 19.4 (IQR 17.6- 21.8) & & & & \\
\hline$>=19.26 \mathrm{~kg} / \mathrm{m}^{2}$ & $51(53.1)$ & $141(49.0)$ & 1 & 1 & 1 & 1 \\
\hline$<19.26 \mathrm{~kg} / \mathrm{m}^{2}$ & $45(46.9)$ & $147(51.0)$ & 0.480 & $1.1(0.7,1.9)$ & - & - \\
\hline \multicolumn{2}{|c|}{ Current BMI kg/m² (median)** 21.6(IQR 18.7- 23.6) } & 21.4 (IQR19.4- 24.0) & & & & \\
\hline$<21.5 \mathrm{~kg} / \mathrm{m}^{2}$ & $51(53.1 \%)$ & $142(49.3 \%)$ & 0.517 & $1.2(0.7,1.8)$ & & \\
\hline$\geq 21.5 \mathrm{~kg} / \mathrm{m}^{2}$ & $45(46.9 \%)$ & $146(50.7 \%)$ & 1 & 1 & 1 & 1 \\
\hline
\end{tabular}

\begin{tabular}{|c|c|c|c|c|c|c|}
\hline \multirow{2}{*}{ Variables } & \multicolumn{2}{|c|}{ First-line ART Failure } & \multicolumn{2}{|c|}{ Bivariate Analysis } & \multicolumn{2}{|c|}{ Multivariate Analysis } \\
\hline & Cases n (\%) & Controls n (\%) & P-value & COR $(95 \% \mathrm{CI})$ & P-value & AOR (95\%CI) \\
\hline Baseline hemoglobin status (median) & 12 (IQR11-12.75) & 12 (IQR11.23-13.08) & & & & \\
\hline$>=12 \mathrm{~g} / \mathrm{dl}$ & $55(57.3)$ & $197(68.4)$ & & 1 & 1 & 1 \\
\hline$<12 \mathrm{~g} / \mathrm{dl}$ & $41(42.7)$ & $91(31.6)$ & 0.047 & $0.6(0.4,1.0)$ & - & - \\
\hline \multicolumn{7}{|c|}{ Current haemoglobin status (median) 13(IQR 12.4-14.1) 13.1(IQR 12.5-14.1) } \\
\hline$>=13.1 \mathrm{~g} / \mathrm{dl}$ & $56(58.3)$ & $200(69.4)$ & 0.046 & $0.6(0.4,1.0)$ & - & - \\
\hline$<13.1 \mathrm{~g} / \mathrm{dl}$ & $40(41.7)$ & $88(30.6)$ & 1 & 1 & 1 & 1 \\
\hline
\end{tabular}

Notes: *Significant at a p-value of $<0.05 * *$ variables which are not candidate by Bivariate logistic regression analysis Notes: ${ }^{\mathrm{b}}$ on campaign session AOR: adjusted odds ratio

\section{Discussion}

This study was identified determinants of first-line ART treatment failure in public hospitals and disclosed that being urban resident, smoking, Khat use, poor adherence, TB coinfection, prior exposure to ART, AZT based medication use and longer duration on HAART enrollment were ascertained to have increased odds of first-line ART failure. In this study, ART users who resided urban appear to have a higher risk of first-line ART failure than those reside rurally. Comparable with a study done in Cameroon which showed that first-line ART treatment failure was less likely to occur among patients residing far from the treatment centers as compared with near the treatment center [40]. This might be due to the good adherence status of patients residing in rural than urban as evidenced by our finding; other assumption could be urban inhabitants might face more HIV-related stigma and discrimination which resulted in poor adherence and failure to take their medication.
With regard to substances use, the present study showed that ART users with a history of smoking were 5.9 times increase the odds of developing first-line ART failure than those who did not; ART users with a history of Khat use were two times more likely to develop first-line ART failure than those who did not. This finding was in tandem with the study findings from Ethiopia [33] and Vietnam [19]. This might be due to the fact that using ARV drugs and substances simultaneously, would prone to mental health problems resulted in poor adherence characterized by failure to take medication properly; moreover interrupting the medication which leads finally to first-line ART treatment failure [34].

ART users with poor medication adherence were two times higher risk of developing first-line ART failure than ART users with good adherence. In line with findings conducted in Ethiopia [8, 14, 35, 41], South Africa [13], Tanzania [12], Rwanda [23] and Kenya [31]. Possibly this might be due to the reality that poor adherence can predispose patients to take medications improperly; moreover, interrupt the therapy and hence allow more viral 
replication which in turn increases infection of more CD4 cells and eventual depletion of their number and hastening to the advanced stage of HIV finally resulted in first-line ART treatment failure.

ART users with TB co-infection appear to have 3.9 times increased risk of developing first-line ART failure than those who did not have TB. Comparable with findings reported from Ethiopia [30, 41] and Nigeria [28]. This might be due to the fact that TB infection impairs cellular immune responses through Tuberculosis-induced apoptosis of CD4 cells which subsequently leads to depletion of CD4 cells and resulting in first-line ART treatment failure [16].

ART users with a history of exposure to any type of ARV regimen prior to ART initiation appear to have 3.8 times higher risk of developing first-line ART treatment failure than those who did not expose. In tandem with the findings from South Africa [13, 22] and Peru [24]. This might be due to poor adherent to ARVs because of the drug's adverse effect [42]. Patients with AZT based medication users have 4.8 times increase the odds of developing first-line ART failure than those with TDF based medication users. Comparable with the study findings from Tanzania [29] and Kenya [31]. This might be due to the fact that AZT induces more adverse effects including severe anemia, nausea, and vomiting that might contribute to reduced treatment adherence [31].

ART users, who were on first-line ART for a longer duration (more than median duration of 73 months) appear to have higher risk of developing first-line ART failure than those who stayed lower duration (less than median duration of 73 months). Comparable with study findings from Ethiopia [35] and Swaziland [26]. This might be due to the fact that being on HAART for longer duration increases the probability of experiencing poor adherence and drug interruption which might be resulted in treatment failure [35]. However, our finding contradicts with study finding from Woldia hospital Ethiopia, which revealed that first-line ART failure was less likely occurred among PLWHA who were on ART for a longer duration (mean duration on ART were 48.4 and 76.5 months for cases and controls, respectively) [3]. This might be due to the difference between the baseline characteristics of the study participants.

A study conducted in Ethiopia revealed that age less than 35 years [35] and older age above 40 years [43] were significant factors for first-line ART failure however in our study age was not found to be significantly associated with first-line ART failure. The study finding from Ethiopia showed that ART users who are male were more likely to experience first-line ART treatment failure as compared to females [8] similar study from Uganda identified that virologic suppression was more likely successful among female patients when compared to male patients [44]. However, in our study sex was not found to be significantly associated with first-line ART failure. But our study was supported by findings from Ethiopia and Tanzania which concluded that the odds of first-line ART treatment failure were not associated with age and sex $[3,11]$.

Being widowed marital status, unemployed occupational status, missed follow up appointment and hemoglobin level were significantly associated with first-line ART treatment failure as reported from Ethiopia [3], Uganda [44], Tanzania [29] and Australia [45]. However, in our study, none of these factors were found to be significantly associated with firstline ART failure.

\section{Study limitations}

The present study has some limitations; this study has made use of secondary data were measuring the adherence status of the patients is based on health care providers' assessment, which is inadequate to detect poor adherence even though this method is often in clinical practice in the Ethiopian context. Again, virologic, immunologic or clinical methods were used for the diagnosis of first-line ART treatment failure; however, measuring treatment failure with immunologic or clinical have poor sensitivity and specificity to detect first-line ART treatment failure. Finally, as a case-control study, this study might face recall bias regarding the information given by the study participants.

\section{Conclusion}

Finally, the study revealed that being urban resident, smoking, Khat use, poor baseline adherence, TB coinfection, exposed to any type of ARV prior to ART initiation, taking AZT based initial first-line ARV and 73 months and more duration on HAART were identified to be the independent determinant factors. The findings of this study indicate the need for more dedication and effort from TB/HIV program managers, health care providers and clients to act on the determinant factors so as to sustain the long-term efficacy of HAART, decrease burden of HIV, improve quality of life and hence, achieving the ambitious 90-90-90 UNAIDS target of 2030 of first-line ART treatment failure.

TB/HIV focal persons and health care providers should focus on the assessment and management of medication compliance for urban residents and longer duration ART users. Assessment and management of substance use disorders are highly recommended besides ARV medication refill among patients taking ART. TB/HIV focal persons and health care providers should give attention and dedicate time with PLWHA to provide enhanced adherence counseling and peer support to improve adherence.

Health care providers, as well as TB/HIV program managers, should give attention to early screening and management of tuberculosis infection among PLWHA. It is advisable health care providers should initiate ART with the recommended TDF-based first-line ART regimens instead of AZT-based. Close follow up of adherence and ensuring proper use of ARVs is important to reduce failure of the firstline ART among those who take ARVs for different purposes before being eligible for ART.

\section{List of Abbreviations}

ART: Antiretroviral Therapy; BMI: Body Mass Index; 
D4T: Stavudine; IQR: Inter Quartile Range; PLWHA: People Living with HIV/AIDS; PMTCT: Prevention of Mother-toChild Transmission SSA: Sub-Saharan Africa; TB: Tuberculosis; 3TC: Lamivudine; AIDS: Acquired Immune Deficiency Syndrome; AOR: Adjusted Odds Ratio; ARV: Antiretroviral; AZT/ZDV: Zidovudine; CI: Confidence Interval; EFV: Efavirenz; HAART: Highly Active Antiretroviral Therapy; HIV: Human Immunodeficiency Virus; NNRTI: Non-Nucleoside Reverse-Transcriptase Inhibitor NRTI: Nucleoside Reverse-Transcriptase Inhibitor; NVP: Nevirapine PEP: Post-Exposure Prophylaxis; TDF: Tenofovir Disoproxil Fumarate; UNAIDS: Joint United Nations Program on HIV/AIDS; WHO: World Health Organization.

\section{Declarations}

Ethics approval and consent to participate

Ethical approval was obtained from Jimma University Institute of Health Ethical Review Committee. Written informed consent was obtained from each study participant before conducting the interviews. The confidentiality of information obtained from each study participant was ensured through anonymity. The findings of the study are communicated in an aggregated form where individual information cannot be disclosed in any way.

\section{Availability of Data and Material}

The datasets generated and/or analyzed during the current study are available from the corresponding author on reasonable request.

\section{Competing Interests}

The authors declare that they have no competing interests

\section{Funding}

This study was funded by Jimma University. The views presented in the article are of the author and not necessarily express the views of the funding organization.

\section{Authors' Contributions}

ABA, ANT, LSD, and TS: Involved in the inception, design, data acquisition, analysis, and interpretation, and wrote the manuscript. EKG and TBL: Participated in design, data acquisition and analysis, administrative matters and reviewing the manuscript. MG and DA: Participated in data acquisition, data management, and analysis as well as critically reviewing the manuscript.

\section{Acknowledgements}

The study participants are greatly acknowledged for their cooperation. Research assistants are also acknowledged for tirelessly collecting, supervising and encoding the data. Finally, Jimma University is highly acknowledged for covering the cost of the study.

\section{References}

[1] UNAIDS. Global AIDS Monitoring. Geneva, Switzerland: UNAIDS; 2018.

[2] UNAIDS: Report on the global AIDS epidemic. Geneva, Switzerland: UNAIDS; 2017.

[3] Babo YD, Alemie GA, Fentaye FW. Predictors of first-line antiretroviral therapy failure amongst HIV-infected adult clients at Woldia Hospital, Northeast Ethiopia. PLoS One. 2017; 12 (11): 1-19.

[4] Leng X, Liang S, Ma Y et al. HIV virological failure and drug resistance among injecting drug users receiving first-line ART in China. BMJ Open. 2014; 4 (e005886): 1-8.

[5] WHO: The use of antiretroviral drugs for treating and preventing HIV infection. Recommendations for a Public Health Approach; Geneva, Switzerland: WHO; 2016.

[6] Patrikar S, Shankar S, Kotwal A, Basannar DR, Bhatti V, Verma $\mathrm{R}$, et al. Predictors of first-line antiretroviral therapy failure and burden of second-line antiretroviral therapy. MJAFI. 2017 Jan 1; 73 (1): 5-11.

[7] Subhasish Kamal Guha DM. Management of Failure of Antiretroviral Treatment; 2016.

[8] Haile D, Takele A, Gashaw K, Demelash H, Nigatu D. Predictors of Treatment Failure among Adult Antiretroviral Treatment ( ART ) Clients in Bale Zone Hospitals, South Eastern Ethiopia. PLoS One. 2016; 11 (10): 2-8.

[9] Allam RR, Murhekar M V, Bhatnagar T, Uthappa CK, Nalini C. Predictors of immunological failure and determinants of suboptimal CD4 testing among adults with HIV on first-line antiretroviral therapy in Andhra Pradesh, India, 2008-2011. Trans R Soc Trop Med Hyg. 2017; 109 (March 2015): 32533.

[10] Huong DTM, Bannister W, Phong PT, Kirk O, Peters L, Mih $\mathrm{B}$, et al. Factors associated with HIV-1 virological failure in an outpatient clinic for HIV-infected people in Haiphong, Vietnam. Int J STD AIDS [Internet]. 2011 [cited 2017 Nov 4]; 22 (659): 659-64.

[11] Hawkins C, Ulenga N, Liu E, Aboud S, Mugusi F, Chalamilla $G$, et al. HIV virological failure and drug resistance in a cohort of Tanzanian HIV-infected adults. Journal Antimicrob Chemother. 2016; 71 (10.1093/jac/dkw051): 1966-74.

[12] Anthony Kapesa et al. Determinants of immunological failure among clients on the first-line treatment with highly active antiretroviral drugs in Dar es Salaam, Tanzania. Asian Pac J Trop Biomed. 2014; 4 ((suppl2)): s620-624.

[13] El-khatib Z, Katzenstein D, Marrone G, Laher F, Mohapi L, Morris L, et al. Adherence to Drug-Refill Is a Useful Early Warning Indicator of Virologic and Immunologic Failure among HIV Patients on First-Line ART in South Africa. PLoS One. 2011; 6 (3): 1-10. 
[14] Biset Ayalew et al. First-line antiretroviral treatment failure and associated factors in HIV patients at the University of Gondar Teaching Hospital, Gondar, Northwest Ethiopia. Dove press. 2016; 8 (10.2147/HIV): 141-6.

[15] Gesesew HA, Ward P, Woldemichael K, et al. Immunological failure in HIV-infected adults from 2003 to 2015 in Southwest Ethiopia : a retrospective cohort study. BMJ Open. 2018; 8 (e017413): 1-10.

[16] Assefa et al. The effect of incident tuberculosis on immunological response of HIV patients on highly active antiretroviral therapy at the University of Gondar hospital, northwest Ethiopia: a retrospective follow-up study. BMC Infect Dis. 2014; 14 (468): 1-8.

[17] Melsew YA, Terefe MW, Tessema GA, Ayele TA. AIDS \& Clinical Rate of Immunological Failure and its Predictors among Patients on Highly Active Antiretroviral Therapy at Debremarkos Hospital, Northwest Ethiopia: A Retrospective Follow up Study. J AIDS Clin Res. 2013; 4 (5): 1-4.

[18] Bekelech Bayou et al. Assessment of the magnitude and associated factors of immunological failure among adult and adolescent HIV-infected patients in St. Luke and Tulubolo Hospital, Oromia Region, Ethiopia. PanAfrican Med J. 2015; 21 (291): 1-10.

[19] Pollack TM, Duong HT, Pham TT, Do CD, Colby D. Cigarette smoking is associated with high HIV viral load among adults presenting for antiretroviral therapy in Vietnam. PLoS One. 2017; 12 (3): 1-11.

[20] Ruperez et al. Determinants of virological failure and antiretroviral drug resistance in Mozambique. Journal Antimicrob Chemother. 2017; 70 (9): 2639-47.

[21] Istri $\mathrm{C}$ et al. Factors associated with first-line antiretroviral therapy ( ART ) failure among HIV / AIDS patients at Sanglah Hospital, Bali. Public Heal Prev Med Arch. 2017; 5 (1): 4-11.

[22] Datay et al. Associations With Virologic Treatment Failure in Adults on Antiretroviral Therapy in South Africa. J Acquir Immune Defic Syndr. 2010; 54 (5): 489-95.

[23] J. d. Ndahimana et al. Drug resistance mutations after the first 12 months on antiretroviral therapy and determinants of virological failure in Rwanda. Trop Med Int Heal. 2016 Jul; 21 (7): 928-35.

[24] Jorge et al. Risk factors associated with virologic failure in HIV-infected patients receiving antiretroviral therapy at a public hospital in Peru. PMC. 2014; 30 (1): 42-8.

[25] Matare T, Shambira G, Gombe NT, Tshimanga M, Bangure D et al. Factors Associated with Human Immunodeficiency Virus First-Line Treatment Failure in Zvishavane District, Austin J HIV/AIDS Res. 2015; 2 (1): 1-6.

[26] Jobanputra K, Parker LA, Azih C, Okello V, Maphalala G, Kershberger B, et al. Factors Associated with Virological Failure and Suppression after Enhanced Adherence Counselling, in Children, Adolescents and Adults on Antiretroviral Therapy for HIV in Swaziland. PLoS One. 2015; 10 (2): $1-12$.

[27] Fujie Zhang et al. Predictors of Virologic Failure in HIV-1 Infected Adults Receiving First-Line Antiretroviral Therapy in 8 Provinces in China. Infect Dis Soc Am. 2017; 50 (2): 264 71.
[28] Musa BM, Musa B, Muhammed H, Ibrahim N. Incidence of tuberculosis and immunological profile of TB / HIV coinfected patients in Nigeria. Ann Thorac Med. 2015; 10 (3): 185-92.

[29] Kamugisha E, Chomboko CL, Kabyemera RM, and KB. Prevalence and Predictors of Immunological Treatment Failure among HIV Infected Adults on the First-line Antiretroviral Therapy in Mbeya Region, Tanzania. Mathews J HIV/AIDS. 2018; 3 (1): 1-9.

[30] Getnet Y. Determinants of First-Line Antiretroviral Treatment Failure in Public Hospitals of Addis Ababa, Ethiopia: Unmatched Case-Control Study. J Biol Agric Healthc. 2014; 4 (15): $1-13$.

[31] Kwobah Charles et al. Factors Associated with First - Line Antiretroviral Therapy Failure amongst HIV - Infected African Patients: A Case - Control Study. World J AIDS. 2012; 2 : 271-8.

[32] FMOH: National Guidelunes for comprehensive HIV Prevention, Care and Treatment. Addis Ababa, Ethiopia: FMOH; 2017.

[33] Niemeyer et al. Predictors of antiretroviral therapy failure in an urban HIV / AIDS clinic in Addis Ababa, Ethiopia. Lancet Glob Heal. 2016; 4: S6.

[34] Nuredin Nassir Azmach. Adherence to Antiretroviral Therapy and Associated Factors Among Adult ARV Users in Arba Minch Hospital, Cent African J Public Heal. 2017; 3 (2): 19 26.

[35] Bayu et al. Determinants of virological failure among patients on highly active antiretroviral therapy in the University of Gondar Referral Hospital, Northwest Ethiopia : a case-control study. Dove Med Press. 2017; 9 (3): p153-157.

[36] UNAIDS: 90-90-90 an ambitious treatment target to help end the AIDS epidemic. Geneva, Switzerland: UNAIDS; 2014.

[37] WHO: Patient monitoring guidelines for HIV care and antiretroviral therapy (ART). Switzerland, Geneva: WHO; 2004.

[38] FMOH: National Guidelines for Comprehensive HIV Prevention, Care and Treatment. Addis Ababa, Ethiopia: FMOH; 2014.

[39] Omar YS, Jenkins A, Altena MVR, Tuck H, Hynan C, Tohow A, et al. Khat Use: What Is the Problem and What Can Be Done? Hindawi Publ Corp. 2015; 2015: 1-7.

[40] Meriki HD, Tufon KA, Afegenwi MH, Nyindem BA, Atanga $\mathrm{PN}$, Anong DN, et al. Immuno-haematologic and virologic responses and predictors of virologic failure in HIV-1 infected adults on first-line antiretroviral therapy in Cameroon. Open Access. 2014; 3 (5): 1-11.

[41] Hailu GG, Hagos DG, Hagos AK, Wasihun G, Dejene TA. Virological and immunological failure of HAART and associated risk factors among adults and adolescents in the Tigray region of Northern Ethiopia. PLoS One. 2018; 30 (5): $1-17$.

[42] V Diaz-Brito et al. Post-exposure prophylaxis for HIV infection : a clinical trial comparing lopinavir/ritonavir versus atazanavir each with zidovudine/lamivudine. Int Med Press. 2012; 17 (10): 337-46. 
[43] Teshome W, Assefa A. Predictors of Immunological Failure of Antiretroviral Therapy among HIV Infected Patients in Ethiopia : A Matched Case-Control Study. PLoS One. 2014; 9 (12): $1-13$.

[44] W. Kipp et al. Gender differences in antiretroviral treatment outcomes of HIV patients in rural Uganda. AIDS Care. 2010; $22(3): 271-8$.
[45] Fong Raymond, Cheng Allen C., Vujovic Olga HJF. Factors associated with virological failure in a cohort of combination antiretroviral therapy at Alfred Hospital Australia. CSIRO. 2013; 10 (5): 442-7. 